\title{
Análise da oferta e eficácia da profilaxia a pré-exposição sexual (PrEP) para o enfrentamento do HIV/AIDS no Brasil
}

\author{
Analysis of the offer and effectiveness of prophylaxis to sexual pre-exposure (PrEP) to fight \\ HIV/AIDS in Brazil
}

Análisis de la oferta y efectividad de la profilaxis a la preexposición sexual (PrEP) para combatir el

VIH / SIDA en Brasil

João Marcos Batista Gomes de Araujo ORCID: https://orcid.org/0000-0001-6877-2179 Faculdade Santa Maria, Brasil

Paula Ívina Oliveira Silva Santos

ORCID: https://orcid.org/0000-0003-0312-3463 Faculdade Santa Maria, Brasil E-mail: paulaiviina@hotmail.com

Marina Gentil Heraclio Maia

ORCID: https://orcid.org/0000-0001-9069-9510 Faculdade Santa Maria, Brasil E-mail: marinamaia_mm@hotmail.com Pedro Elias Morato

ORCID: https://orcid.org/0000-0001-5156-818X Suprema Faculdade de Ciências Médicas e da Saúde de Juiz de Fora, Brasil E-mail: pedroeliasmorato@gmail.com

Maria Lidivânia Batista Gomes ORCID: https://orcid.org/0000-0001-5073-9557 Faculdade Santa Maria, Brasil E-mail: marialidivaniabg@hotmail.com

José Cândido da Silva Nóbrega ORCID: https://orcid.org/0000-0002-0976-3763 Universidade Federal de Campina Grande, Brasil E-mail: jcandidosn@uol.com.br

Manoel Marques de Souto Nóbrega Filho ORCID: https://orcid.org/0000-0003-4512-2733

Faculdade de Ciências Médicas da Paraíba

E-mail: manoelmarquesnobrega@gmail.com

\begin{abstract}
Resumo
A epidemia do vírus do HIV/AIDS é considerado um fenômeno de largas proporções, e por seu caráter pandêmico e gravidade, representa um grande problema de saúde pública, carecendo de políticas mais humanizadas e eficientes, e nesse sentido, um dos métodos mais recentes de profilaxia a pré-exposição sexual, trata-se da (PrEP) que é um tratamento precoce farmacológico feito através da combinação de dois medicamentos, que devem ser tomados diariamente pelo usuário antes do mesmo ter a infecção pelo vírus. Dessa forma, o presente artigo propõe uma análise sobre os desafios da oferta e eficácia da PrEP para o enfrentamento do HIV no Brasil. Para tanto, foi realizada uma revisão teórica de literatura exploratória sobre a temática através da utilização do método de abordagem dedutivo, com a utilização de dados bibliográficos de natureza qualitativa através da pesquisa documental. A partir disso, concluiu-se que através do uso da profilaxia, foi possível reduzir o risco das pessoas adquirir a infecção pelo vírus, pois essa estratégia mostrou-se bastante eficaz e segura em pessoas com risco aumentado de adquirir a infecção, entretanto, se torna importante que as políticas públicas de enfrentamento ao HIV/AIDS no país sejam amplamente divulgadas a fim de atingir a maioria do público que está vulnerável ao contágio pelo vírus.
\end{abstract}

Palavras-chave: Saúde pública; PrEP; HIV. 


\begin{abstract}
The HIV/AIDS virus epidemic is considered a phenomenon of large proportions and, due to its pandemic character and severity, represents a major public health problem, lacking more humanized and efficient policies, and in this sense, one of the mos recent methods of prophylaxis to sexual pre-exposure, this is (PrEP) which is an early pharmacological treatment made through the combination of two drugs, which must be taken daily by the user before he has the virus infection. Thus, this article proposes a reflection on the challenges of the offer and effectiveness of PrEP to fight HIV in Brazil. Therefore, a theoretical review of exploratory literature on the subject was carried out using the deductive approach method, with the use of bibliographic data of a qualitative nature through documentary research. From this, it was concluded that through the use of prophylaxis, it was possible to reduce the risk of people acquiring the virus infection, as this strategy proved to be quite effective and safe in people at increased risk of acquiring the infection, however, if makes it important that public policies to fight HIV/AIDS in the country are widely publicized and in order to reach the majority of the public that is vulnerable to contagion by the virus.
\end{abstract}

Keywords: Public health; Prep; HIV.

\title{
Resumen
}

La epidemia del virus del VIH / SIDA es considerada un fenómeno de grandes proporciones y, por su carácter y gravedad pandémica, representa un gran problema de salud pública, careciendo de políticas más humanizadas y eficientes, y en este sentido, uno de los métodos de profilaxis más recientes. a la preexposición sexual, esto es (PrEP) que es un tratamiento farmacológico precoz que se realiza mediante la combinación de dos fármacos, los cuales deben ser tomados diariamente por el usuario antes de contraer la infección por el virus. Así, este artículo propone una reflexión sobre los desafíos de la oferta y la efectividad de la PrEP para combatir el VIH en Brasil. Por lo tanto, se realizó una revisión teórica de la literatura exploratoria sobre el tema utilizando el método de enfoque deductivo, con el uso de datos bibliográficos de carácter cualitativo a través de la investigación documental. A partir de esto, se concluyó que mediante el uso de profilaxis, era posible reducir el riesgo de que las personas adquirieran la infección por el virus, ya que esta estrategia demostró ser bastante efectiva y segura en personas con mayor riesgo de contraer la infección, sin embargo, si hace importante que las políticas públicas de lucha contra el VIH / SIDA en el país sean ampliamente difundidas y con el fin de llegar a la mayoría de la población vulnerable al contagio por el virus.

Palabras clave: Salud pública; Prep; VIH.

\section{Introdução}

A partir da proliferação da epidemia do vírus da HIV causador da AIDS no mundo, grandes questões que afligem a todos vieram à tona, como a garantia dos direitos humanos, qualidade de vida, políticas de medicamentos e propriedade industrial, por seu caráter pandêmico e sua gravidade, esse vírus representa um dos maiores problemas de saúde pública da atualidade (Parker \& Camargo, 2000).

Dentre os métodos de combate ao vírus da imunodeficiência humana (HIV), a Profilaxia Pré-Exposição (PrEP) foi adotada como uma estratégia de prevenção muito poderosa que consiste no uso diário de um medicamento antirretroviral por pessoas não infectadas pelo HIV, e seu uso correto reduz em mais de $90 \%$ o risco de aquisição da infecção, vale destacar também que o Brasil é pioneiro na América Latina e Caribe ao adotar a PrEP como política pública (Portugal, 2018).

Nesse contexto o presente artigo propõe fazer uma análise sobre os desafios da oferta e da eficácia da profilaxia a préexposição sexual (PrEP) para o enfrentamento do HIV no Brasil, no sentido de averiguar como se deu a inclusão na esfera das políticas de saúde oferecidas para esse público específico.

Essa pesquisa trata-se de um estudo teórico oriundo das reflexões, revisões de literatura e análise de dados de pesquisas, que ajudam a esclarecer a problemática, sob diferentes perspectivas referentes às políticas públicas que são disponibilizadas para o combate ao HIV/AIDS no Brasil e a forma de como esses pacientes responde aos tratamentos.

Sob essa perspectiva, essa pesquisa traz em seu escopo a seguinte preocupação: O Brasil é conhecido mundialmente pela grande eficácia na campanha de combate ao vírus HIV/AIDS e dessa forma oferece através do SUS novos tratamentos precoces como o PrEP, e a partir do uso dessa nova combinação de medicação para o combate ao vírus, será que esse novo tratamento está tendo boa aceitação e eficácia em relação aos usuários? 
Dessa forma, o presente estudo tem como objetivo geral analisar a eficácia do tratamento precoce contra o HIV através do método de profilaxia a pré-exposição sexual (PrEP) no Brasil, aliado aos seguintes objetivos específicos: averiguar por meio de uma breve contextualização histórica os índices e números da epidemia de HIV/AIDS no Brasil e avaliar a eficácia do (PrEP) como forma de tratamento das pessoas com exposição ao vírus no país.

Buscando validar essa afirmação, essa pesquisa foi desenvolvida através da utilização do método de abordagem dedutivo, o qual analisará através de pesquisas realizadas com pacientes de HIV/AIDS a aceitação e eficácia do tratamento do uso de PrEP no Brasil, também será feita uma análise exploratória, através da pesquisa de dados bibliográficos de natureza qualitativa, a qual utilizou-se da análise de artigos, teses, revistas, dissertações, livros e pesquisas, e por fim foi utilizado o método procedimento documental que foi feito por meio de análise de leis e projetos.

Para uma melhor compreensão, esse estudo organiza-se em primeiro lugar em fazer uma abordagem sobre a epidemia do vírus da HIV no Brasil, se faz necessário também, realizar uma análise sobre os tratamentos que são oferecidos no Brasil para o combate desse vírus, e por fim será feita uma síntese sobre a utilização dos PrEPS como forma de prevenção as pessoas que são mais vulneráveis ao contagio do HIV.

\section{Contextualização Histórica sobre a Epidemia de HIV/AIDS no Brasil}

A descoberta da síndrome da imunodeficiência adquirida conhecida como a AIDS em 1981, tornou-se um marco na história mundial, ademais, esse vírus tem destaque entre as grandes enfermidades infecciosas emergentes pela sua imensa magnitude e extensão dos danos causados às populações e, desde a sua origem, cada uma de suas características e repercussões tem sido exaustivamente estudada pela comunidade científica e discutida pela sociedade em geral (Brito, Castilho, \& Szwarcwald, 2001).

Outrossim, o período entre 1981 e 1984 marcou a forma como a AIDS chegou no Brasil, e não foi pelo relato dos casos de pessoas acometidas por essa doença que foram descobertos no país, mais sim, pelo relato da doença pela mídia, e assim, como em outros países a mídia teve um papel fundamental em tornar aquela doença desconhecida como pública (Brasil, 2021).

Nesse contexto, a epidemia da infecção pelo vírus do HIV/AIDS não são problemas apenas de ordem nacional, e sim, constitui fenômeno global, dinâmico e instável, traduzindo-se por verdadeiro mosaico de sub-epidemias regionais, e tem em sua disseminação ser resultante das profundas desigualdades da sociedade brasileira, a propagação da infecção pelo desse vírus, revela epidemia de múltiplas dimensões que vem sofrendo transformações epidemiológicas significativas (Brito, Castilho, \& Szwarcwald, 2001).

Em 1985 foi iniciada a primeira resposta brasileira à AIDS, quando o estado, a sociedade civil e a comunidade cientifica se associaram com um único objetivo comum de fortalecer a criação do SUS em 1988, que foi imprescindível para implementar o programa nacional de Controle das DST/AIDS, baseado nos direitos humanos, visando equidade, inclusão, integralidade e contra o preconceito e a discriminação sendo que o Brasil foi um dos primeiros países a disponibilizar, já em 1996, na saúde pública, tratamento adequado para todas as PVHA (Greco, 2016).

Essa infecção provocada pelo vírus do HIV causador da AIDS é considerada uma epidemia de grave proporção e apresenta diversos fatores de risco associados, como idade, gênero, etnia, orientação sexual e renda média, e no Brasil, de acordo com dados disponibilizados entre os anos de 2007 até junho de 2016, no sistema do Ministério da Saúde ocorreu um amento de 136.945 casos de infecção pelo HIV, entretanto, acredita-se que uma relevante fração de indivíduos infectados pelo vírus não foi identificada e, portanto, não foi reportada para os órgãos de regulação (Brasil, 2016). 
De acordo com dados divulgados do Departamento de Doenças de Condições Crônicas e Infecções Sexualmente Transmissíveis do site do Ministério da Saúde, informações de pesquisa realizada no ano de 2020, constam que foram contabilizadas cerca de 920 mil pessoas vivem com HIV no Brasil, dessas, 89\% foram diagnosticadas, 77\% fazem tratamento com antirretroviral e $94 \%$ das pessoas em tratamento não transmite o HIV por via sexual por terem atingido carga viral indetectável, em 2020, até outubro, cerca de 642 mil pessoas estavam em tratamento antirretroviral, em 2018 eram 593.594 pessoas em tratamento (Brasil, 2020).

Os resultados desses indivíduos que fazem tratamentos com antirretrovirais pelo SUS no Brasil são promissores, e depende exclusivamente da dedicação e adesão dos pacientes aos tratamentos que são disponibilizados através das políticas públicas de combate ao vírus do HIV/AIDS.

\section{Tratamento da HIV/AIDS no Brasil}

O Brasil é reconhecido mundialmente por ter um dos melhores programas de adoção de políticas públicas controversas e bem sucedidas para conter o avanço da epidemia de AIDS no país, e devido a essa integração de ações de tratamento e prevenção foram obtidos resultados importantes como a redução da mortalidade, morbidade, hospitalizações, e aumento da expectativa de vida das pessoas que vivem com HIV/AIDS no país (Fonseca, 2008).

Em consonância o Ministério da Saúde adverte que o maior número de adeptos que fazem adesão a um tratamento está relacionado diretamente à aceitação e à integração de determinado regime terapêutico no cotidiano das pessoas em tratamento, o qual depende principalmente da participação do paciente na tomada de decisões sobre ele.

Concomitante, o comportamento do usuário com o médico em relação ao tratamento possui grande relevância no sucesso da terapia contra o HIV, pois deve haver uma relação de confiança entre o paciente e o médico, no qual o tratamento deve ser entendido em toda a sua amplitude, e refere-se a adesão do usuário quanto a prescrição médica, posologia, à quantidade de medicamentos por horário, tempo de tratamento e às recomendações especiais para determinados medicamentos (Cardoso, 2019).

Dessa forma, torna-se extremamente necessário a adesão do paciente ao esquema terapêutico anti-retroviral (ARV) tendo se tornado esse atualmente, um dos maiores desafios para o sucesso do tratamento do HIV/AIDS e ao mesmo tempo essa é uma das mais poderosas armas contra a AIDS (Silva, 2009).

Em busca de reduzir os efeitos da imunossupressão foi incorporado ao tratamento a Terapia Antirretroviral (TARV) na década de 90, o que provocou aumento da qualidade e do tempo de vida dos pacientes, pois esse tratamento e composto por três antivirais combinados com diferentes mecanismos de ação, considerando a eficácia, efetividade, toxicidade e comodidade posológica, entretanto, esse tratamento também pode provocar uma série de reações adversas como distúrbios metabólicos, que reduzem a adesão ao tratamento(Finkelstein et al., 2015).

Diante das informações, os serviços do Sistema Único de Saúde (SUS) brasileiro dos 26 estados e do Distrito Federal passaram a oferecer à população o tratamento de Profilaxia Pré-Exposição ao HIV (PrEP) e de Profilaxia Pós-Exposição (PEP) a partir do partir do mês de maio de 2018 o que estabeleceu um marco de grande importância no combate à doença no país (Dorin et al., 2021).

É importante salientar que esses tratamentos já foram oferecidos anteriormente em caráter piloto em algumas cidades do país e são recomendados pela Organização Mundial da Saúde (OMS) desde 2012 conforme noticiado pelo Departamento de Doenças Crônicas e Infecções Sexualmente Transmissíveis (DCCI) do Ministério da Saúde (Brasil, 2017). 
Em suma, as políticas de tratamentos que são disponibilizados para o combate ao HIV no Brasil, são reconhecidos mundialmente por terem um dos melhores programas de distribuição e eficácia, porém é preciso que os usuários juntamente com os médicos façam os protocolos de forma continua para que se tenha melhores resultados.

\section{A utilização dos PrEPS como Forma de Prevenção do HIV}

Um dos métodos mais recentes de profilaxia ao HIV, segundo informações disponíveis on-line pelo Departamento de Doenças de Condições Crônicas e Infecções Sexualmente Transmissíveis (DCCI), trata-se da PrEP que é um tratamento precoce farmacológico feito através da combinação de dois medicamentos, que devem ser tomados diariamente pelo usuário antes do mesmo ter a infecção pelo vírus HIV, os que são denominados como soronegativos, a PrEP não deve ser tomada por pessoas que já estejam acometidos pela infecção do vírus HIV (Dorin et al., 2021).

Nessa conjectura, a profilaxia a pré-exposição sexual (PrEP) está em constante debate e surge como uma nova possibilidade de prevenção ao HIV, e em consequência tem aumentando a expectativa acerca do controle da epidemia mundial, pois, essa profilaxia caracteriza-se pelo uso cotidiano da combinação de dois antirretrovirais (tenofovir associado à entricitabina - TDF/FTC) que são utilizados antes das práticas sexuais, com grau de proteção de 96\% nas relações sexuais, quando utilizada por pelo menos quatro dias na semana (Anderson, 2011).

Com resultados promissores, a profilaxia pré-exposição sexual (PrEP) vem sendo considerada estratégica e promissora no controle da epidemia de HIV mundialmente, entretanto, é necessário que esse conhecimento acumulado pelos estudos de eficácia, devem ser amplamente divulgados principalmente para as populações mais vulneráveis à infecção, de forma a alcançar uma ampla cobertura da PrEP (Zucchi, 2018).

Dessa forma, foram apontadas algumas considerações acerca de uma relativa incompatibilidade entre a estrutura existente nos serviços e as diretrizes do Ministério da Saúde para oferta de PrEP e nessa perspectiva foi identificado que o maior êxito da PrEP como política pública de saúde depende de dois aspectos centrais: assegurar que os serviços sejam ambientes culturalmente diversos e livres de discriminação e a intensificação das intervenções comunitárias, incluindo as redes sociais, de forma a reduzir iniquidades no acesso aos serviços e à PrEP (Zucchi, 2018).

Foram coletados e divulgados dados de monitoramento da Profilaxia Pré-Exposição - PrEP no Brasil pelo site do DCCI que abrange coleta de dados entre 01/01/2018 e 28/02/2021, e foi constatado que nesse período houve 18.704 usuários ativos de PrEP, e no tocante ao perfil dos usuários, o maior número da faixa etária de pessoas se deu entre 30 e 39 anos (41\% do total), com maior taxa de adesão de homens cis gays/homens que fazem sexo com homens (HSH), representando 15.457 usuários $(82,6 \%$ do total), seguido por mulheres cisgênero $(7,8 \%)$, homens hétero cisgênero $(6,0 \%)$, mulheres trans $(0,4 \%)$, travestis $(0,4 \%)$ e homens trans $(0,3 \%)$. Quanto a distribuição dos usuários pelo critério de raça/cor, 57,2\% dos usuários são brancos/amarelos, 42,4\% são negros e 0,3\% indígenas (Brasil, 2021).

Ao todo no ano de 2020 foi constatado a adesão de 12.855 novos usuários de PrEP no país e somados apenas os meses de janeiro e fevereiro do ano de 2021, houve 2.704 novos usuários neste período de dois meses ( Dorin et al., 2021).

Ainda segundo dados divulgados pelo Ministério da Saúde através do boletim epidemiológico HIV/AIDS de 2017, dessa pesquisa, foi constatado que no país a epidemia de HIV/AIDS possui concentração em alguns grupos populacionais específicos, os quais representam a maioria dos casos da infecção, como gays, homens que fazem sexo com homens, pessoas trans e profissionais do sexo, ademais, houve um crescimento significativo da infecção pelo HIV em adolescentes e jovens (Brasil, 2017). 
Em conclusão, é perceptível que os indivíduos que fazem uso de PrEPS no Brasil em sua grande maioria são grupos muito específicos formado por gays, mulheres cis, homens e pessoas trans profissionais do sexo, dessa forma é preciso que se tenha estratégias de políticas de acesso à informação sobre a eficácia desse tratamento que seja divulgada de forma mais abrangente, principalmente para um maior alcance deste grupo.

\section{Resultados e Discussão}

Segundo Foner (2016), foram encontrados excelentes resultados da eficácia da Profilaxia Pré-Exposição (PrEP) ao HIV que atestaram a segurança do tratamento em inúmeros estudos clínicos que foram realizados em vários subgrupos de populações, o qual sua efetividade foi evidenciada em estudos de demonstração.

É possível constatar ainda que, a importância do uso de antirretrovirais como o PrEP no Brasil se dá devido às altas taxas da eficácia do uso desses medicamentos como forma de controle do aumento de incidência de casos de HIV, e nesse sentido é importante que as políticas públicas de enfrentamento ao vírus sejam amplamente divulgadas a fim de atingir a maioria do público com pré disposição ao acometimento pelo vírus do HIV.

Nesse contexto, a política brasileira de enfrentamento ao HIV/AIDS tem conhecimento de que as políticas de intervenção e prevenção que são oferecidas de forma isolada, não são eficazes para a redução de novas infecções, pois a transmissão e infecção operam de forma dinâmica em diferentes camadas sociais da população, e dessa forma, devem ser desenvolvidas estratégias de combate a disseminação desse vírus (Brasil, 2018).

Portanto, essas estratégias devem ser ofertadas de forma mais abrangentes, para que se possa garantir uma maior diversidade de opções que oriente as decisões dos indivíduos assistidos, ademais, é preterível que essas pessoas possam escolher os métodos que melhor se adequem às suas condições e circunstâncias de vida, tendo como princípios norteadores a garantia de direitos humanos e o respeito à autonomia e integridade desses indivíduos (Brasil, 2018).

Em síntese, é de relevante importância que os serviços de saúde que oferecem o tratamento com PrPES no Brasil sejam amplamente divulgados para que as pessoas que precisam desse tratamento precoce não tenham estigmas ou medo de inicia o tratamento para que seja reduzido o maior número de indivíduos infectados por HIV no Brasil.

\section{Considerações Finais}

A profilaxia a pré-exposição ao HIV, que consiste no uso de antirretrovirais para reduzir o risco de adquirir a infecção pelo vírus, tem se mostrado bastante eficaz e segura em pessoas com risco aumentado de adquirir a infecção, outrossim, e esse tratamento com antirretrovirais para o combate do vírus, outrossim, é importante ressaltar que esse tratamento é oferecido pelo SUS como política de saúde para os indivíduos que tem uma predisposição ao contágio do HIV/AIDS devido a sua vulnerabilidade do convívio com pessoas acometidas pelo vírus.

E a partir dessa perspectiva, dados divulgados de pesquisas realizadas nos anos de 2018 e 2021 no país, desenharam o perfil do público especifico que faz uso do tratamento com PrEP, que é composto em sua grande maioria de homens cis gays/homens que fazem sexo com homens, representando 82,6 \% do total, seguido por mulheres cisgênero, homens hétero cisgênero, mulheres trans, travestis e homens trans representado por pessoas brancas, negras e indígenas.

O presente artigo, no entanto, não se propõe a apontar caminhos pré-estabelecidos, ou mesmo encontrar respostas para todos os seus questionamentos, mas sim, através de uma discussão teórica, problematizar temáticas de relevância social e que possuem desdobramentos diretos na vida dos indivíduos. 
Research, Society and Development, v. 10, n. 12, e22101219095, 2021

(CC BY 4.0) | ISSN 2525-3409 | DOI: http://dx.doi.org/10.33448/rsd-v10i12.19095

Porém, é preciso que se tenha maior conhecimento da alta taxa de eficácia do uso de PrEP como forma de controle de aumento de incidência de HIV, a qual se torna importante a divulgação das políticas públicas de enfrentamento ao HIV/AIDS no país para que sejam amplamente divulgadas e fim de atingir a maioria do público que está vulnerável ao contágio pelo vírus.

\section{Referências}

Anderson, P. L., Kiser, J. J., Gardner, E. M., Rower, J. E., Meditz, A., \& Grant, R. M. (2011). Pharmacological considerations for tenofovir and emtricitabine to prevent HIV infection. J Antimicrob Chemother 66:240-50.

Brasil. Ministério da Saúde. (2020). Boletim Epidemiológico sobre a doença aponta queda na taxa de detecção de Aids no país desde 2012. http://www.aids.gov.br/pt-br/noticias/casos-de-aids-diminuem-no-brasil.

Brasil. Ministério da Saúde. (2018). Secretaria de Vigilância em Saúde. Departamento de Vigilância, Prevenção e Controle das Infecções Sexualmente Transmissíveis, do HIV/Aids e das Hepatites Virais. Protocolo Clínico e Diretrizes Terapêuticas para Profilaxia Pré-Exposição (PrEP) de Risco à Infecção pelo HIV. Brasília. http://www.aids.gov.br/pt-br/pub/2017/protocolo-clinico-e-diretrizes-terapeuticas-para-profilaxia-pre-exposicao-prep-de-risco.

Brasil. Ministério da Saúde. (2017). Nota Informativa N007/2017 - DDAHV/SVS/MS. http://azt.aids.gov.br/documentos/siclom_operacional/Nota\% 20Informativa\%20007\%20-\%20protocolo\%20de\%20uso\%20ARV\%20-\%202017.pdf

Brasil. Ministério da Saúde. (2016). Boletim Epidemiológico Aids e DST, V, n. 1. www.aids.gov.br/system/tdf/pub/2016/59427/bole tim_2016_1_pdf_16375.pdf?file=1\&type=node\&id=59427\&force=1.

Brasil. Ministério da Saúde. (2015). Departamento de Doenças de Condições Crônicas e Infecções Sexualmente Transmissíveis. Boletim Epidemiológico HIV/AIDS. Brasília. ano 4, n. 1, 2015. http://www.aids.gov.br/pt-br/pub/2015/boletim-epidemiologico-hivaids.

Brasil. Ministério da Saúde. (2008). Programa Nacional de DST e AIDS: Manual de adesão ao tratamento para pessoas vivendo com HIV e AIDS. Brasília. http://bvsms.saude.gov.br/bvs/publicacoes/manual_adesao_tratamento.

Brito, M. A., Castilho, A. E., \& Szwarcwald, L. C. (2001). AIDS e infecção pelo HIV no Brasil: uma epidemia multifacetada artigo de Opinião. Rev. Soc. Bras. Med. Trop. 34(2) abr. https://doi.org/10.1590/S0037-86822001000200010.

Cardoso, G. P., \& Arruda, A. (2019). As representações sociais da soropositividade e sua relação com a observância terapêutica. Rev. Ciênc Saúde 14; 10(1). http://www.scielo.br/pdf/csc/v10n1/a16v10n1

Dorin, L. D., Silva, T. B., \& Rentes Medeiros, D. (2021). Prevenção ao HIV no Brasil: Representações sociais, estigma e desafios dos usuários da PrEP. Pathos: Revista Brasileira de Práticas Públicas e Psicopatologia, 7(1), ISSN 2447-6137.

Finkelstein, J. L., Gala, P., Rochford, R., Glesby, M. J., \& Mehta, S. (2015). HIV/AIDS and lipodystrophy: implications for clinical management in resourcelimited settings. J Int AIDS Soc. 18:19033. https://doi.org/10.7448/IAS.18.1.19033.

Foner, V. A. et al. (2016). Effectiveness and safety of oral HIV pre-exposure prophylaxis for all populations: a systematic review and meta-analysis. AIDS, [S.1.], 30(12).

Fonseca, E. M., (2008). Resposta brasileira à epidemia de HIV/AIDS entre usuários de drogas injetáveis: marcos, avanços e desafios. 139 f. Tese (Doutorado em Saúde Pública) - Escola Nacional de Saúde Pública

Arouca, S. Fundação Oswaldo Cruz. https://www.arca.fiocruz.br/handle/icict/4499.

Grant, R. M. et al. (2017). Preexposure chemoprophylaxis for HIV prevention in men who have sex with men. The New England Journal of Medicine, [S.1.], 363(27). https://doi.org/10.1056/ NEJMoa1011205

Greco, B. D. (2016). Trinta anos de enfrentamento à epidemia da Aids no Brasil, 1985-2015 Ciênc. saúde colet. 21(5). https://doi.org/10.1590/141381232015215.04402016 .

Parker, R., \& Camargo, K. R. Jr. (2000). Pobreza e HIV/AIDS: aspectos antropológicos e sociológicos. Cadernos de Saúde Pública, 16(Supl. 1).

Portugal, J. (2018) Lancet HIV destaca os resultados do PrEP Brasil. Fiocruz, Comunicação e Informação. Recuperado de https://portal.fiocruz.br/noticia/lancet-hiv-destaca-osresultados-do-prep-brasil

Silva, A. L. C. N., Waidman, M. A. P., \& Marcon, S. S. (2009). [Adhesion And Non Adhesion To Anti-Retroviral Therapy: The Two Faces Of A Same Experience]. Rev Bras Enferm. 62(2):213-20. HTTPS://DOI.ORG/10.1590/S0034-71672009000200007.

Zucchi, E. M. et al., (2018). Da evidência à ação: desafios do Sistema Único de Saúde para ofertar a profilaxia pré-exposição sexual (PrEP) ao HIV às pessoas em maior vulnerabilidade. Cad. Saúde Pública 34(7). https://doi.org/10.1590/0102-311X00206617. 